\title{
Structural and stability characteristics of jets in crossflow - CORRIGENDUM
}

\author{
D. R. Getsinger, L. Gevorkyan, T. Shoji, O. I. Smith and A. R. Karagozian
}

\author{
doi:10.1017/jfm.2014.605, Published by Cambridge University Press, \\ 7 November 2014 \\ doi:10.1017/jfm.2016.5, Published by Cambridge University Press, \\ 2 February 2016
}

\begin{abstract}
After extensive examination and testing, we have determined that erroneous jet flow rates were used in some of the experiments in the above-noted papers (Getsinger et al. 2014; Gevorkyan et al. 2016). This error only pertains to those experiments involving acetone planar laser-induced fluorescence (PLIF) imaging, in which the jet fluid is a mixture of helium, nitrogen and acetone vapour. For these acetone PLIF experiments, the flow rates of helium and nitrogen were prescribed in a manner that erroneously neglected the contribution of acetone vapour to the combined mixture flow rate. Therefore, our actual jet flow rates were higher than those stated in the two papers for such experiments. The identification of instabilities cited (convective instability versus absolute instability in the upstream shear layer, for example) is unchanged in the papers, as are the overall findings on mixing metrics and trends as well as jet structure. The overarching conclusions for the papers are also unchanged. However, many of the parameter values cited in the figures require correction.

To correct the research record, we note the following for transverse jet experiments in the papers mentioned above involving acetone PLIF experiments only:
\end{abstract}

(i) For equidensity transverse jet experiments, where the jet-to-crossflow density ratio $S$ is unity, the jet Reynolds numbers should be increased by $22 \%$. In most cases, this alters $R e_{j}$ from the stated value of 1900 to a corrected value of 2300 . Likewise, momentum flux ratios $J$ should be increased by $48 \%$. For example, the range of conditions explored should be altered as follows:

\begin{tabular}{cc}
\hline Original $J$ value & Corrected $J$ value \\
2 & 3 \\
5 & 7 \\
8 & 12 \\
10 & 15 \\
12 & 18 \\
20 & 30 \\
30 & 44 \\
41 & 61 \\
\hline
\end{tabular}

Some or all of the above-noted parameter values appear in Getsinger et al. (2014) in figures 6-9, 11 and 12, as well as in Gevorkyan et al. (2016) for equidensity conditions in parts of figures 4-22. 
(ii) For low-density transverse jets, at $S=0.55$, the jet Reynolds number should be increased by $16 \%$, and $J$ values by $34 \%$. These corrections for $S=0.55$ apply to Getsinger et al. (2014) in figure 13 and to Gevorkyan et al. (2016) in figures 7, 11,15 and 19 . For the low-density case $S=0.35$, there was no apparent error in the flow rate, and the values of the parameters are correct as stated in the papers. The same can be said for results from simultaneous PLIF/particle image velocimetry experiments in Getsinger et al. (2014).

(iii) Figures comparing jets with different density ratios did not actually have matched momentum flux ratios $J$, as desired; this applies to figure 13 in Getsinger et al. (2014) and to figures 7, 11, 15, 19 and 21 in Gevorkyan et al. (2016). Nevertheless, the overall conclusions in the papers regarding the influence of lowered densities on jet structure, instabilities and the contribution of crossflow entrainment to mixing are still correct and consistent with additional experimental datasets (Gevorkyan 2015).

\section{Acknowledgements}

The authors are deeply indebted to E. Harris and A. Besnard of the University of California, Los Angeles (UCLA) for discovering this discrepancy and taking additional data to verify and correct these parameter values.

\section{REFERENCES}

Getsinger, D. R., Gevorkyan, L., Smith, O. I. \& Karagozian, A. R. 2014 Structural and stability characteristics of jets in crossflow. J. Fluid Mech. 760, 342-367.

GEVORKYAN, L. 2015 Structure and mixing characterization of variable density transverse jet flows. $\mathrm{PhD}$ thesis, University of California, Los Angeles (UCLA), CA.

Gevorkyan, L., Shoji, T., Getsinger, D. R., Smith, O. I. \& Karagozian, A. R. 2016 Transverse jet mixing characteristics. J. Fluid Mech. 790, 237-274. 